\title{
Spatial and Temporal Eye-Hand Coordination Relies on the Parietal Reach Region
}

\author{
Eun Jung Hwang, ${ }^{1,2}$ Markus Hauschild, ${ }^{1}$ Melanie Wilke, ${ }^{1,3,4}$ and Richard A. Andersen ${ }^{1}$ \\ ${ }^{1}$ Division of Biology and Biological Engineering, California Institute of Technology, Pasadena, California 91125, ${ }^{2}$ Division of Biological Sciences, University \\ of California, San Diego, California 92093, ${ }^{3}$ Department of Cognitive Neurology, University of Göttingen, Göttingen, 37075, Germany, and ${ }^{4}$ German Primate \\ Center, Leibniz Institute for Primate Research, Göttingen, 37077, Germany
}

\begin{abstract}
Coordinated eye movements are crucial for precision control of our hands. A commonly believed neural mechanism underlying eyehand coordination is interaction between the neural networks controlling each effector, exchanging, and matching information, such as movement target location and onset time. Alternatively, eye- hand coordination may result simply from common inputs to independent eye and hand control pathways. Thus far, it remains unknown whether and where either of these two possible mechanisms exists. A candidate location for the former mechanism, interpathway communication, includes the posterior parietal cortex (PPC) where distinct effector-specific areas reside. If the PPC were within the network for eye-hand coordination, perturbing it would affect both eye and hand movements that are concurrently planned. In contrast, if eye-hand coordination arises solely from common inputs, perturbing one effector pathway, e.g., the parietal reach region (PRR), would not affect the other effector. To test these hypotheses, we inactivated part of PRR in the macaque, located in the medial bank of the intraparietal sulcus encompassing the medial intraparietal area and area $5 \mathrm{~V}$. When each effector moved alone, PRR inactivation shortened reach but not saccade amplitudes, compatible with the known reach-selective activity of PRR. However, when both effectors moved concurrently, PRR inactivation shortened both reach and saccade amplitudes, and decoupled their reaction times. Therefore, consistent with the interpathway communication hypothesis, we propose that the planning of concurrent eye and hand movements causes the spatial information in PRR to influence the otherwise independent eye control pathways, and that their temporal coupling requires an intact PRR.
\end{abstract}

Key words: inactivation; movement endpoints; PPC; reaches; reaction time; saccades

\section{Introduction}

In daily manual tasks including simple reaches and grasps, people look at task-relevant objects before acting on them (Johansson et al., 2001; Hayhoe et al., 2003; Ko et al., 2010). The preceding foveation of task-relevant objects provides high-acuity visual information about the position and shape of target objects, enhancing the precision of the following hand movement (Enright, 1995; Johansson et al., 2001; Kato and Fukuda, 2002; Crawford et al., 2004). Consequently, reach errors increase in a gazedependent manner when reach and gaze are spatially decoupled (e.g., reaching for coffee while reading a newspaper; Henriques and Crawford, 2000). Eye-hand coupling also manifests as high

Received Aug. 30, 2013; revised Aug. 8, 2014; accepted Aug. 13, 2014.

Author contributions: E.J.H., M.H., and R.A.A. designed research; E.J.H., M.H., and M.W. performed research; E.J.H. analyzed data; E.J.H., M.W., and R.A.A. wrote the paper.

This work was supported by NIH Grants EY007492, EY013337, EY005522, and DARPA award N66001-10-C-2009; E.J.H. was supported by NIH Career Development Award K99 NS062894; and M.W. was supported by the Hermanand Lilly Schilling Foundation and the German Research Foundation (DFG) WI 406/1-1. We thank Drs Igor Kagan and James Bonaiuto for the acquisition and processing of MR images, and Dr Bardia Behabadi for scientific discussion.

The authors declare no competing financial interests.

Correspondence should be addressed to Dr Eun Jung Hwang, Division of Biology and Biological Engineering, California Institute of Technology, 1200 East California Boulevard, MC 216-76, Pasadena, CA 91125. E-mail: eunjung@caltech.edu.

DOI:10.1523/JNEUROSCI.3719-13.2014

Copyright $\odot 2014$ the authors $\quad 0270-6474 / 14 / 3412884-09 \$ 15.00 / 0$ trial-by-trial correlations between reach and saccade reaction times (Sailer et al., 2000; Song et al., 2008; Dean et al., 2011).

The spatial and temporal eye-hand coupling suggests commonly shared information between the neural pathways underlying the control of eye and hand movements (Frens and Erkelens, 1991; Sailer et al., 2000; Flanagan et al., 2008). The common information may arise simply from common inputs from the shared sensory and cognitive processes regarding stimulus location and movement onset. Additionally and/or alternatively, the common information could form if the two pathways exchange and match the spatial and temporal information. $\mathrm{Al}$ though direct evidence is lacking, the posterior parietal cortex (PPC) has been proposed to be a place for such interpathway communication, and crucial for eye-hand coordination (Carey, 2000; Van Donkelaar et al., 2000; Gaveau et al., 2008; Dean et al., 2012).

In both human and macaque PPC, distinct areas are selectively activated for different effector movements (Culham, 2006; Filimon, 2010; Vesia and Crawford, 2012; Konen et al., 2013; Andersen et al., 2014). In the macaque PPC, the parietal reach region (PPR) and the lateral intraparietal area (LIP) are involved in the control of reaches and saccades, respectively (Snyder et al., 1997; Andersen and Cui, 2009; Hwang et al., 2012; Yttri et al., 2013). However, when both effector movements are concurrently planned, those effector-specific areas may communicate 
Table 1. The average number of trials per target location, task condition, and session

\begin{tabular}{|c|c|c|c|c|c|c|c|}
\hline \multirow[b]{3}{*}{ Monkey } & \multirow{3}{*}{$\begin{array}{l}\text { Session } \\
\text { no. }\end{array}$} & \multicolumn{6}{|c|}{ №. of trials per target } \\
\hline & & \multicolumn{2}{|l|}{ Eye-hand } & \multicolumn{2}{|c|}{ Hand-only } & \multicolumn{2}{|c|}{ Eye-only } \\
\hline & & Control & Inactivation & Control & Inactivation & Control & Inactivation \\
\hline \multirow[t]{7}{*}{ Y } & 1 & $22(21)$ & $11(10)$ & & & 22 & 11 \\
\hline & 2 & $20(20)$ & $19(17)$ & & & 14 & 19 \\
\hline & 3 & $21(20)$ & $22(18)$ & & & 20 & 22 \\
\hline & 4 & $13(12)$ & $25(21)$ & & & 14 & 25 \\
\hline & 5 & $25(25)$ & $25(25)$ & 25 & 29 & 26 & 27 \\
\hline & 6 & $25(24)$ & $25(23)$ & 28 & 33 & 26 & 25 \\
\hline & 7 & $26(24)$ & $13(12)$ & 40 & 13 & 26 & 15 \\
\hline \multirow[t]{6}{*}{ G } & 1 & $8(7)$ & $19(17)$ & 9 & 21 & 9 & 19 \\
\hline & 2 & $15(12)$ & $18(6)$ & 14 & 18 & 13 & 14 \\
\hline & 3 & $19(15)$ & $11(8)$ & 18 & 9 & 17 & 9 \\
\hline & 4 & $15(12)$ & $19(17)$ & 13 & 18 & 13 & 17 \\
\hline & 5 & $18(17)$ & $19(17)$ & 17 & 20 & 18 & 18 \\
\hline & $6^{*}$ & $258(234)$ & $266(240)$ & 33 & 53 & 34 & 57 \\
\hline
\end{tabular}

Each session is a pair of control and inactivation that were typically separated by $1 \mathrm{~d}$. The numbers in parenthesis indicate the number of trials in which both eye and hand positions at the onset of go cue were within $1 \mathrm{~cm}$ from the hand-fixation target. The italicized sessions and tasks are data previously presented in Hwang et al. (2012).

*Tasks were performed with only two targets (contralateral and ipsilateral). In the other sessions, tasks were performed with six targets.

and coordinate with each other either within PPC or through downstream cortical areas such as the frontal cortex (BattagliaMayer et al., 2006).

Suggestive of information transmission from the saccade controller LIP (and/or its downstream areas) to reach controllers, inactivating LIP increases both reach and saccade reaction times when both effectors move concurrently to the same remembered location (Yttri et al., 2013). Furthermore, a subpopulation of LIP neurons predict the reaction times of concurrently planned reaches and saccades (Dean et al., 2012). These LIP neurons are coherent with the beta band local field potentials (LFPs), which also predict the reaction time of concurrent reaches and saccades in both LIP and PRR (Dean et al., 2012; Hagan et al., 2012). Thus, coherent spiking activity in LIP and PRR may be a mediator between the two pathways to temporally coordinate the concurrent movements of the two effectors.

However, information transmission from reach to saccade controllers has not been shown. Hence, this study examines how inactivating the reach control area PRR affects eye-hand coordination.

\section{Materials and Methods}

Two male adult monkeys (Macaca mulatta) were tested. The California Institute of Technology Institutional Animal Care and Use Committee approved the animal procedures, which were performed in accordance with NIH guidelines.

\section{Behavioral tasks}

The monkeys sat in a dark room $\sim 40 \mathrm{~cm}$ in front of an LCD monitor mounted behind a touch-sensitive screen, and performed center-out reach and/or saccade movements in their frontoparallel plane. Both monkeys used the arm contralateral to the inactivated hemisphere (left arm for Monkey Y, right for Monkey G). Eye position was tracked with an infrared eye tracker (ISCAN, $120 \mathrm{~Hz}$ ). For a subset of data, the continuous hand position was also recorded using an optical motion tracking system (Northern Digital, $2 \mathrm{kHz}$ ).

Monkey Y performed seven pairs of control and inactivation sessions, and Monkey G performed six pairs (Table 1). A control session paired with each inactivation session typically occurred $1 \mathrm{~d}$ before or after inactivation (8 pairs with control on the previous day of inactivation, 4 on the next day, 1 after $7 \mathrm{~d}$ ). In most sessions, the monkeys performed all three tasks, the eye-hand, hand-only, and eye-only tasks described below, except four control and inactivation sessions in which Monkey Y performed the eye-hand, and eye-only but not hand-only task. Data in 11 pairs ( 6 in Monkey Y and 5 in Monkey G) were collected from the same experiments presented in Hwang et al. (2012).

Eye-hand task. In this task, the eyes were not constrained in any way so that the monkeys would show natural eye-hand coordination. A trial began as the monkeys touched the central hand-fixation target (Fig. 1a). After $0.5 \mathrm{~s}$, a target stimulus (a green circle) was presented at 1 of 2 or 6 evenly spaced points around an invisible circle (radius $7.26 \mathrm{~cm}, \sim 10.5^{\circ}$ for Monkey Y, and $8.25 \mathrm{~cm}, \sim 11.9^{\circ}$ for Monkey G). After $0.3 \mathrm{~s}$, the target stimulus was extinguished, and an at least $1 \mathrm{~s}$ (uniformly sampled between 1 and $1.3 \mathrm{~s}$ ) long memory period followed. The memory period ended as the central hand-fixation target was extinguished, cueing the monkeys to move the hand ("go" cue). If the monkeys initiated a reach movement within $2 \mathrm{~s}$ from the go cue and the movement ended within a tolerance window from the target, they received a drop of juice $0.3 \mathrm{~s}$ after the movement ended. The reach endpoint tolerance was $4 \mathrm{~cm}$ in radius for both monkeys. The same tolerances for reaction times and endpoints were used in both control and inactivation conditions. Note, however, the monkeys have been trained in the tasks described in this study for several months with a smaller tolerance window $(\sim 2 \mathrm{~cm})$ before the current study began.

Hand-only task. This task was identical to the eye-hand task except for the following: two superimposed central fixation targets, a triangle for hand and a square for eyes, were simultaneously presented. The monkeys reached to the remembered target after the central hand-fixation target was extinguished while keeping the eyes fixated on the central eyefixation target.

Eye-only task. This task was the same as the hand-only task except for the following: the target was a red square. After the central eye-fixation target was extinguished, the monkeys moved the eyes to the remembered target while keeping the hand on the central hand-fixation target. The saccade endpoint tolerance was $\sim 7^{\circ}(5 \mathrm{~cm})$ for Monkey Y and $\sim 9^{\circ}(6.5$ $\mathrm{cm}$ ) for Monkey G.

The tolerances were set leniently to observe the behavioral consequences of the inactivation while suppressing error-based adaptations and to keep the monkeys motivated by minimizing the number of failed trials. Different task and target location trials were randomly interleaved. The average number of successful trials per target and task condition in each session is shown in Table 1 . In general, $\sim 20$ successful trials (range, $8-266)$ per target and task condition were completed in each session.

\section{Behavioral data analysis}

We first measured the onset, offset, start point and endpoint of each movement. Reach onset was when the hand was lifted off from the touchsensitive screen, and offset was when the hand touched the screen again. Saccade onset and offset were the first time when eye velocity fell below $\sim 37^{\circ} \mathrm{s}$ for $5 \mathrm{~ms}$ when going backward and forward in time from peak velocity. The movement start-point and endpoint position were the effector positions just before onset and just after offset.

Using these measures, we computed the following movement parameters: the reaction time is the time elapsed from the go cue until movement onset. The movement duration is the time between onset and offset. The movement amplitude is the Euclidian distance between a movement start point and an endpoint. To minimize the variance of movements due to variance of initial conditions, our analysis for movement amplitudes included trials in which both the eye and hand start points were within $1 \mathrm{~cm}$ from the central fixation targets. This criterion was satisfied by $95 \%$ of control and $90 \%$ of inactivation trials in Monkey Y, and $89 \%$ of control and $83 \%$ of inactivation trials in Monkey G (Table 1).

Correlation analysis. A trial-by-trial correlation between any two parameters was computed using the Pearson correlation coefficient. We also computed a normalized correlation coefficient for each session by computing a Pearson correlation coefficient for every five consecutive trials and taking the mean of all the consecutively computed coefficients. This normalization process resolves the issue that the total number of trials varied across sessions (Table 1). It can also resolve the issue that a few outliers can dominate the Pearson correlation coefficient because the 
a

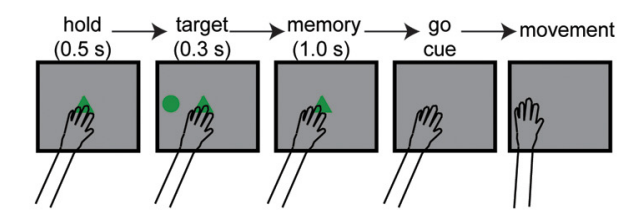

C

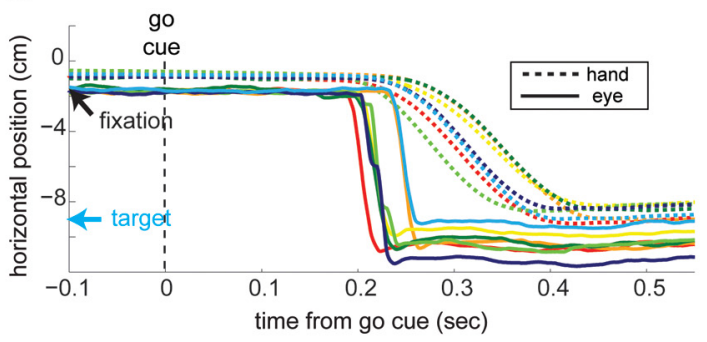

b

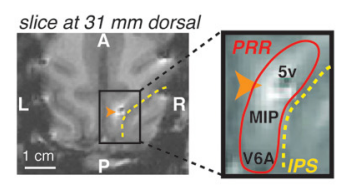

monkey G

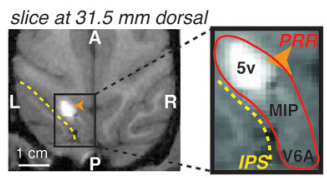

d

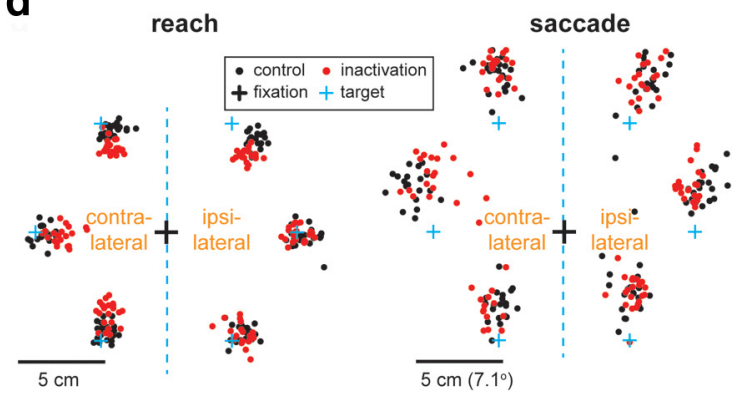

Figure 1. Natural eye-hand coordination in the eye-hand task. $\boldsymbol{a}$, The temporal sequence of events in the eye-hand task. Eyes were unconstrained. $\boldsymbol{b}$, The inactivation site shown on the horizontal brain MRI slice. The bright spot indicates where MR visible gadolinium was injected in the same way as muscimol. c, The hand and eye positions of seven example trials in the eye- hand task are aligned to the go cue. Each pair of a solid and a dashed line in the same color represents the hand and eye positions of the same single trial. The arrows mark the positions of the central hand-fixation and the reach target. $\boldsymbol{d}$, Reach and saccade endpoints for six target locations in the eye-hand task from an example pair of a control and an inactivation session. The saccades occurred under free-gaze condition in the absence of experimental requirements.

majority of five-trial windows do not include outliers. In addition, the normalization process can reduce the correlation caused by slowly varying global states, such as arousal and motivation, because those states are likely steady within short five-trial windows.

Assessment of inactivation effects. Inactivation effects on any movement parameter such as reaction time and movement amplitude were assessed at four different levels. At all levels, statistical tests were applied to each animal and each target separately. First, we assessed the inactivation effect at a global level by comparing two pools, each containing all control and inactivation trials, respectively. From each pool, we computed the global mean and its $95 \%$ confidence interval using a bootstrap (10,000 times sampling). Then, the significance level of the difference in the global mean between the two global pools was measured using a null distribution computed from shuffling trials between the two pools 10,000 times (permutation test). Second, if the global means were significantly different between control and inactivation in both animals, we assessed the robustness of inactivation effects across sessions. To do so, we measured the inactivation effect for each inactivation session by computing the mean of each inactivation session (within-session mean) and comparing it to the mean of its counterpart control session (within-pair difference). The significance level of the within-pair difference in a pair of a control and an inactivation session was estimated using a null distribution computed from shuffling control and inactivation trials within the pair 10,000 times (permutation test). Third, we assessed the significance of the inactivation effects, treating paired control and inactivation sessions as matched samples, by applying the Wilcoxon signed rank test (WSRT). Last, we compared within-session means across control versus inactivation sessions using the Wilcoxon rank sum test (WRST), treating the two pools as independent samples. The significance level $p<0.05$ is used throughout the paper and it was adjusted using Bonferroni correction for multiple comparisons over the six target locations when initially exploring the inactivation effects using global means.

\section{Reversible inactivation}

A majority of data presented in this paper was collected from the same experiments as in Hwang et al., 2012 (Table 1), in which a detailed description of the reversible inactivation procedure is available. In brief, we identified a PRR region in each monkey through extracellular recording experiments before the inactivation experiments. PRR includes a number of reach selective cortical areas, extending from the dorsal aspect of the parieto-occipital area ( $\mathrm{PO}$ ) including V6A and the posterior aspect of the medial bank of the intraparietal sulcus (IPS) including the medial intraparietal area (MIP) and extending anteriorly in the IPS into area $5 \mathrm{~V}$ (Andersen et al., 2014). We selected an area in the medial bank of the IPS as the inactivation site where we found most neurons were active selectively during planning and execution of memory-guided reaches as opposed to saccades. The same site was inactivated in all inactivation sessions. Gadolinium injection in this constant location showed that our inactivation was contained within the medial wall of the mid-posterior portion of the IPS, including areas MIP and $5 \mathrm{~V}$ (Fig. $1 b$ ). In each inactivation session, a stainless steel beveled-tip cannula (28-30 gauge, Plastic One) was acutely lowered to the target location in PRR. Then, typically 5 $\mu \mathrm{l}$ (range, 3.5-10) of muscimol solution $(5 \mathrm{mg} / \mathrm{ml}, \mathrm{pH} \sim 7.4$ ) was injected at $1 \mu \mathrm{l} / \mathrm{min}$. The behavioral experiment began $35-60 \mathrm{~min}$ after the injection started and lasted up to $3 \mathrm{~h}$. In five of the control sessions, we performed sham control by injecting saline solution instead of muscimol. Because the saline control sessions produced similar results as nonsaline control sessions, we combined all control sessions together.

\section{Results}

Effects of PRR inactivation on spatial eye-hand coordination In the eye-hand task (Fig. 1a), the monkeys reached the remembered location of a visual cue, with their eyes unconstrained. In this free-gaze condition, the monkeys' gaze was within $1 \mathrm{~cm}$ $\left(\sim 1.4^{\circ}\right)$ from the central hand-fixation target at the onset of the go cue signal in $99 \%$ of control trials in Monkey $\mathrm{Y}$, and $96 \%$ of control trials in Monkey G (Fig. 1c). The central eye fixation was not required, but expected given that the go cue signal was the extinction of the central hand-fixation target. Following the go cue, the monkeys naturally, again without experimentally imposed requirements, made a saccade toward the remembered reach target in nearly all trials (Fig. $1 c, d$ ). The saccades directed toward the reach target were observed in $98 \%$ of control trials in Monkey Y, and $97 \%$ in Monkey G.

A similar eye behavior was observed in inactivation trials. In 99\% and 95\% of inactivation trials in Monkeys Y and G, their gaze was within $1 \mathrm{~cm}$ from the fixation target at the go cue onset. These ratios are not significantly different from those of control trials ( $\chi^{2}$ test, $p=0.49$ for Monkey Y and $p=0.33$ for Monkey $\mathrm{G})$. In addition, the spatially coupled saccades following the go cue were observed in $96 \%$ of inactivation trials in Monkey Y, and 
a
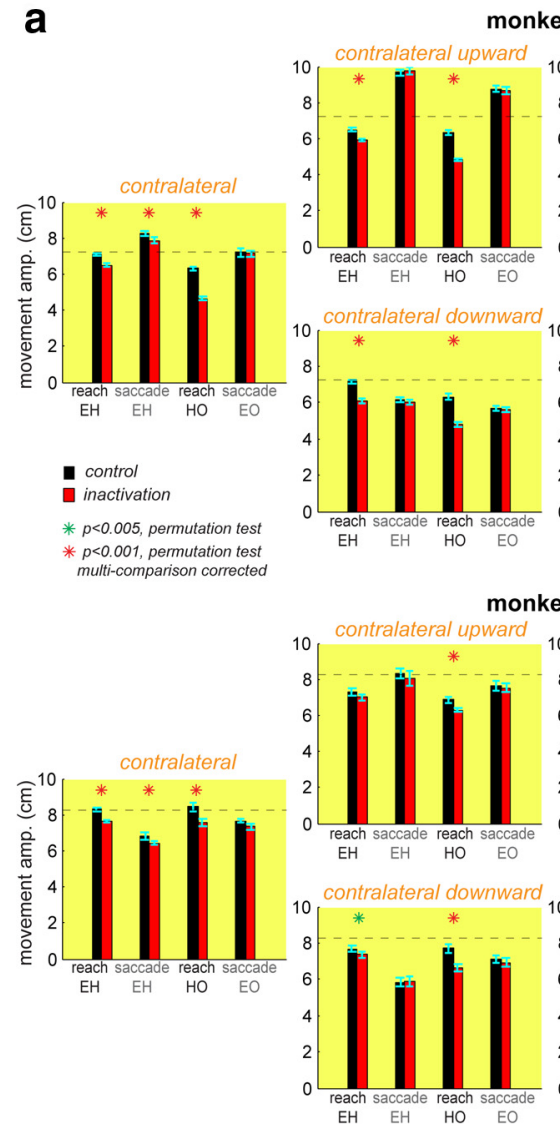

monkey $\mathbf{Y}$

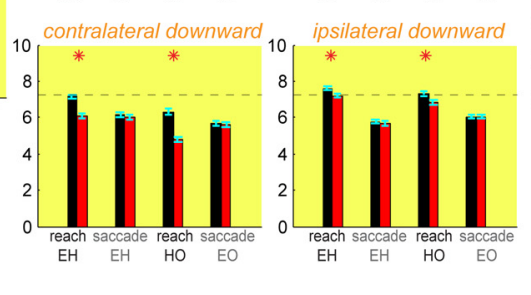

monkey G

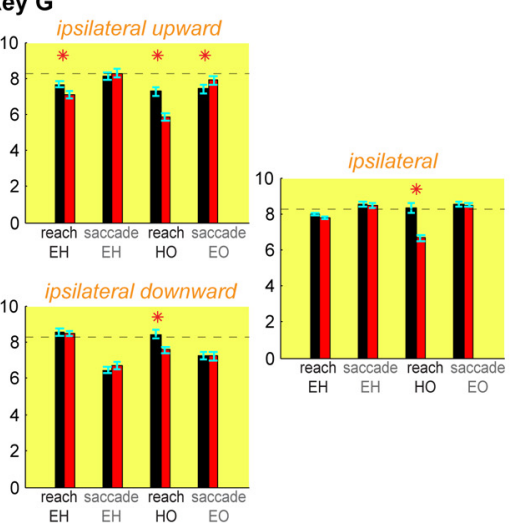

b
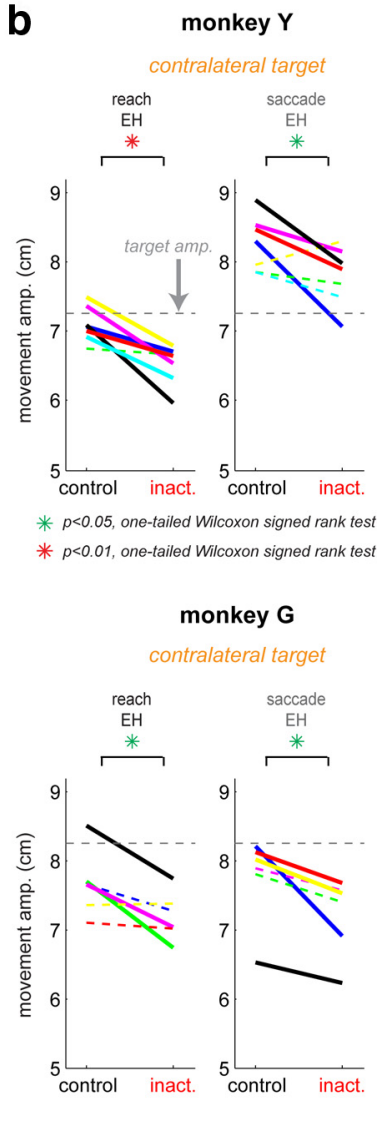

Figure 2. PRR inactivation shortens the amplitude of coordinated saccades and reaches but not of saccades executed alone. $\boldsymbol{a}$, Reach and saccade amplitudes in three task conditions for each of six target locations. For each target, the mean amplitude and its $95 \%$ confidence interval are plotted for control versus inactivation reaches in the eye- hand task, control versus inactivation saccades in the eye- hand task, control versus inactivation reaches in the hand-only task, and control versus inactivation saccades in the saccade-only task, from left to right. The horizontal dashed line in each plot marks the target amplitude. $\boldsymbol{b}$, Within-session means of reach and saccade amplitudes in the eye-hand task for the contralateral target, in control versus inactivation sessions. Each pair of a control and an inactivation session is connected with a solid line if their means are significantly different $(p<0.05)$, and with a dashed line otherwise.

$96 \%$ in Monkey G, which is not significantly different from control trials $\left(\chi^{2}\right.$ test, $p=0.57$ for Monkey Y and $p=0.88$ for Monkey $G)$. Therefore, PRR inactivation did not influence the frequency with which the eyes were fixated on the central handfixation target before the go cue, or the frequency with which spatially coupled reaches and saccades occurred.

However, PRR inactivation altered the endpoints of spatially coupled reaches and saccades, changing movement amplitudes for some targets. Figure $1 d$ displays reach and saccade endpoints with the eye-hand task from an example pair of a control and an inactivation session. For the contralateral horizontal target, both the reach and saccade endpoints in inactivation are clustered more closely to the central hand-fixation target compared with the control. As a result, both reach and saccade amplitudes for this contralateral target were shorter for inactivation than control. Note that in the eye-hand task, only a reach to the target was required, and a saccade to the reach target was not necessary for the monkey to receive a reward. The lack of requirement for eye movements may explain why the saccade endpoints were more scattered than the reach endpoints. In comparison, in the eye-only task in which monkeys were specifically required to move the eyes to a remembered cue location, saccades were as accurate as reaches (Hwang et al., 2012).

To quantify the inactivation effects on reach and saccade amplitude in the eye-hand task, we combined all control versus inactivation trials from all sessions, and compared the global means of the two pools for each target location (Fig. 2a). The significance of the global mean difference between control and inactivation was assessed using a permutation test with trial shuffling (see Materials and Methods). In Monkey Y, the reach amplitude was significantly smaller for inactivation than control for all six targets, whereas the saccade amplitudes were significantly smaller for inactivation only for the two horizontal targets (Table 2 shows means, SEs, and $p$ values of the statistical tests for each target). In Monkey $G$, the reach amplitude decreased significantly for three targets (contralateral, contralateral downward, and ipsilateral upward), whereas the saccade amplitude decreased significantly only for the contralateral target (Table 2). Thus, in both monkeys, PRR inactivation caused a significant reduction of reach and saccade amplitudes in the eye-hand task for the contralateral target.

These results stand in contrast to the inactivation effects on reach and saccade amplitudes when each effector moved alone (Fig. 2a; Table 2). The reach amplitude in the hand-only task was significantly reduced by PRR inactivation for all targets in both monkeys. The saccade amplitude in the eye-only task was not significantly different between control and inactivation for any target but one in Monkey $G$ in which the saccade amplitude slightly increased. Therefore, unlike the eye-hand task, saccade amplitude was not reduced by the saccade only task.

To examine the robustness of the inactivation effects for the contralateral target in the eye-hand task, we computed the mean 
Table 2. Summary statistics of inactivation effects on movement amplitude, reaction time, and movement duration (from top to bottom)

\begin{tabular}{|c|c|c|c|c|c|c|c|c|c|}
\hline & \multicolumn{4}{|c|}{ Changes in mean (mean, SEM, $p$ ) } & \multicolumn{4}{|c|}{ Changes in variance (mean, SEM, $p$ ) } & \multirow{2}{*}{$\begin{array}{l}\text { Changes in CC } \\
\text { (mean, SEM, } p \text { ) }\end{array}$} \\
\hline & Reach_EH & Saccade_EH & Reach_HO & Saccade_E0 & Reach_EH & Saccade_EH & Reach_HO & Saccade_E0 & \\
\hline \multicolumn{10}{|c|}{$\begin{array}{l}\text { Movement } \\
\quad \text { amplitude }\end{array}$} \\
\hline Monkey Y:I & -0.260 .0920 .000 & -0.210 .0830 .001 & -0.630 .0800 .000 & -0.070 .0751 .000 & -0.040 .0701 .000 & -0.060 .0881 .000 & -0.020 .0481 .000 & 0.210 .0900 .001 & -0.240 .1090 .012 \\
\hline IU & -0.380 .0900 .000 & -0.010 .1471 .000 & -1.010 .0520 .000 & 0.240 .1790 .370 & -0.020 .0771 .000 & -0.580 .3210 .092 & -0.050 .0441 .000 & -0.950 .9071 .000 & -0.070 .1121 .000 \\
\hline CU & -0.570 .0650 .000 & 0.110 .1491 .000 & -1.460 .0810 .000 & -0.080 .1251 .000 & -0.320 .0820 .000 & 0.340 .3611 .000 & -0.270 .0590 .005 & 0.850 .4520 .002 & -0.170 .1260 .294 \\
\hline$C$ & -0.590 .0680 .000 & -0.380 .1230 .000 & -1.680 .0790 .000 & -0.060 .1481 .000 & 0.130 .0720 .092 & 0.200 .2581 .000 & 0.050 .0821 .000 & -1.380 .8860 .180 & 0.270 .1350 .066 \\
\hline$C D$ & -1.070 .0860 .000 & -0.130 .0990 .335 & -1.600 .1040 .000 & 1.111 .2371 .000 & 0.130 .0800 .851 & 0.070 .1091 .000 & -0.050 .1341 .000 & -0.070 .1571 .000 & -0.040 .1841 .000 \\
\hline ID & -0.440 .0930 .000 & -0.080 .0850 .986 & -0.490 .1040 .000 & -0.020 .0741 .000 & 0.050 .0891 .000 & 0.000 .0981 .000 & -0.220 .0950 .077 & 0.020 .0711 .000 & -0.020 .1131 .000 \\
\hline Monkey G: I & -0.120 .0820 .232 & -0.080 .0911 .000 & -1.570 .1900 .000 & 0.050 .0911 .000 & -0.140 .0740 .040 & 0.190 .5201 .000 & -0.450 .1840 .137 & -0.010 .0841 .000 & -0.010 .0781 .000 \\
\hline IU & -0.640 .1430 .000 & 0.120 .1581 .000 & -1.780 .1520 .000 & 0.430 .1550 .001 & 0.260 .1500 .202 & 0.090 .2101 .000 & -0.190 .2081 .000 & -0.290 .2581 .000 & 0.310 .2520 .528 \\
\hline CU & -0.220 .1460 .237 & -0.230 .2351 .000 & -0.520 .1070 .000 & -0.160 .1791 .000 & 0.180 .1460 .545 & 1.310 .8760 .156 & -0.150 .1270 .565 & -0.150 .2711 .000 & -0.040 .2351 .000 \\
\hline$C$ & -0.690 .0760 .000 & -0.440 .1040 .000 & -0.710 .1650 .000 & -0.080 .2961 .000 & -0.210 .0810 .007 & -1.871 .4460 .007 & -0.630 .2540 .033 & 0.330 .1430 .016 & 0.170 .0780 .048 \\
\hline$C D$ & -0.310 .1350 .005 & 0.050 .1841 .000 & -1.030 .1520 .000 & -0.150 .1470 .856 & 0.180 .1701 .000 & 0.530 .3470 .199 & -0.150 .1621 .000 & 0.160 .1651 .000 & 0.000 .2211 .000 \\
\hline ID & -0.110 .1241 .000 & 0.200 .1290 .214 & -0.850 .1390 .000 & 0.030 .1531 .000 & -0.280 .1540 .053 & 0.020 .1281 .000 & -0.270 .1880 .657 & 0.290 .1840 .139 & -0.220 .1940 .564 \\
\hline \multicolumn{10}{|l|}{ Reaction time } \\
\hline Monkey Y: I & 2.744 .1331 .000 & 3.753 .8991 .000 & 24.249 .1280 .001 & -0.224 .3551 .000 & -0.840 .5050 .095 & 0.940 .6020 .094 & -1.511 .2630 .662 & 0.390 .6651 .000 & 0.020 .1231 .000 \\
\hline IU & -3.244 .1471 .000 & 3.494 .8501 .000 & 30.308 .4870 .000 & -15.066 .0490 .004 & -0.400 .3130 .468 & 1.550 .7800 .013 & -1.310 .9330 .411 & -1.381 .1880 .912 & -0.200 .1080 .048 \\
\hline CU & 0.503 .4881 .000 & -8.184 .3990 .058 & 17.906 .9320 .002 & 7.275 .0730 .247 & -0.360 .1980 .073 & -1.210 .6150 .051 & -0.750 .5540 .579 & 1.380 .9820 .258 & 0.160 .1330 .516 \\
\hline$C$ & 16.026 .2040 .000 & 16.547 .9250 .019 & 9.086 .6460 .394 & -16.395 .5980 .000 & 2.712 .2110 .750 & 0.831 .2131 .000 & -0.830 .9811 .000 & -1.731 .2470 .336 & -0.260 .1090 .018 \\
\hline$C D$ & 12.865 .1460 .000 & -18.0411 .5280 .156 & 28.456 .4400 .000 & -4.785 .2481 .000 & 1.531 .7491 .000 & 0.941 .0221 .000 & -0.660 .8601 .000 & -1.110 .8150 .462 & -0.080 .2001 .000 \\
\hline ID & 2.576 .4431 .000 & -22.357 .0710 .000 & -1.2113 .7761 .000 & -7.814 .1630 .046 & -4.904 .4321 .000 & -1.120 .6440 .128 & -1.873 .7321 .000 & 0.460 .7381 .000 & 0.090 .1761 .000 \\
\hline Monkey G: I & -9.074 .8590 .058 & -24.723 .8460 .000 & -32.012 .590 .001 & -9.135 .8780 .165 & -1.360 .6650 .025 & -0.330 .5791 .000 & -4.984 .6140 .683 & 0.200 .7281 .000 & 0.040 .1151 .000 \\
\hline IU & 27.8117 .4500 .161 & 0.545 .9161 .000 & 2.3312 .681 .000 & 5.718 .6781 .000 & 7.757 .6531 .000 & -0.300 .4681 .000 & -6.623 .9360 .008 & 1.481 .3240 .750 & 0.040 .1361 .000 \\
\hline CU & -6.1713 .9191 .000 & -8.569 .4041 .000 & 16.7413 .0450 .451 & -19.036 .8610 .001 & -2.772 .0740 .423 & -0.170 .7311 .000 & 4.023 .3890 .488 & -0.220 .4751 .000 & -0.280 .2020 .684 \\
\hline$C$ & -24.425 .5040 .000 & -15.833 .6730 .000 & -10.5311 .501 .000 & -9.824 .9690 .027 & 1.681 .7451 .000 & -0.910 .3810 .001 & 5.144 .2540 .654 & -0.670 .2490 .000 & -0.150 .0720 .036 \\
\hline$C D$ & 32.6119 .2490 .110 & 30.908 .9900 .000 & 9.5411 .7431 .000 & -1.597 .4501 .000 & 14.526 .8870 .005 & 1.360 .6750 .056 & 1.171 .5381 .000 & -0.320 .7291 .000 & 0.120 .1891 .000 \\
\hline ID & 14.1924 .0941 .000 & -5.387 .3061 .000 & 1.2312 .4141 .000 & -10.828 .1540 .356 & 2.496 .2241 .000 & 0.190 .5961 .000 & 0.462 .6341 .000 & 0.891 .0871 .000 & 0.100 .1751 .000 \\
\hline \multicolumn{10}{|l|}{$\begin{array}{l}\text { Movement } \\
\text { duration }\end{array}$} \\
\hline Monkey Y:I & -16.74 .430 .000 & 0.40 .551 .000 & -15.74 .380 .000 & 0.40 .531 .000 & -0.030 .4491 .000 & 0.000 .0041 .000 & -0.070 .3261 .000 & 0.000 .0051 .000 & 0.090 .1401 .000 \\
\hline IU & -22.55 .450 .000 & 0.10 .591 .000 & -28.74 .340 .000 & 0.80 .640 .622 & -1.100 .8540 .668 & 0.000 .0051 .000 & -0.460 .3040 .805 & -0.010 .0070 .051 & 0.030 .0951 .000 \\
\hline CU & -28.95 .640 .000 & 0.30 .871 .000 & -22.84 .820 .000 & 0.00 .961 .000 & -3.451 .1960 .000 & -0.010 .0090 .501 & -0.170 .4721 .000 & -0.010 .0101 .000 & 0.080 .1131 .000 \\
\hline$C$ & -24.45 .170 .000 & 1.00 .810 .418 & 1.110 .411 .000 & -0.20 .991 .000 & -1.390 .7060 .043 & 0.010 .0090 .311 & 5.315 .5511 .000 & 0.030 .0391 .000 & -0.030 .1631 .000 \\
\hline$C D$ & -16.03 .790 .000 & 2.21 .250 .060 & 44.022 .440 .014 & -1.51 .160 .404 & -0.250 .1710 .506 & 0.070 .0640 .196 & 38.7713 .9220 .000 & 0.010 .0130 .835 & -0.070 .2061 .000 \\
\hline $\mathrm{ID}$ & -14.04 .800 .000 & 0.80 .600 .504 & -10.54 .740 .016 & 2.11 .080 .039 & -2.031 .2900 .016 & 0.000 .0061 .000 & -0.520 .3620 .550 & 0.010 .0120 .557 & -0.130 .1391 .000 \\
\hline Monkey G: I & -34.45 .960 .000 & -3.61 .580 .006 & -38.711 .320 .000 & -1.60 .880 .076 & -5.134 .6651 .000 & 0.140 .2171 .000 & -3.573 .7231 .000 & -0.030 .0210 .428 & 0.060 .0541 .000 \\
\hline IU & -8.07 .550 .829 & -0.80 .740 .763 & -13.09 .950 .383 & 1.60 .920 .095 & 0.430 .7991 .000 & 0.010 .0080 .139 & -0.554 .3821 .000 & 0.000 .0111 .000 & 0.270 .1410 .132 \\
\hline CU & 3.19 .811 .000 & -1.61 .240 .425 & 1.26 .901 .000 & -1.81 .320 .302 & -2.252 .6211 .000 & -0.040 .0400 .959 & -0.210 .3971 .000 & -0.020 .0291 .000 & -0.270 .1790 .282 \\
\hline$C$ & -41.34 .250 .000 & -0.80 .520 .145 & -20.07 .230 .000 & -0.40 .871 .000 & -1.710 .9580 .076 & -0.020 .0160 .151 & 2.051 .4060 .257 & 0.020 .0241 .000 & -0.100 .0650 .354 \\
\hline$C D$ & 2.410 .061 .000 & -2.61 .240 .023 & 8.99 .071 .000 & -2.01 .290 .151 & 0.192 .3391 .000 & -0.010 .0141 .000 & 1.992 .1201 .000 & -0.020 .0120 .271 & 0.060 .2041 .000 \\
\hline ID & -7.38 .021 .000 & 0.21 .131 .000 & -10.66 .240 .099 & -1.11 .010 .869 & -0.680 .7921 .000 & 0.010 .0120 .623 & -0.270 .2560 .844 & 0.000 .0131 .000 & 0.220 .1570 .780 \\
\hline
\end{tabular}

The left column indicates Monkeys Y and G targets (I, ipsilateral; IU, ipsilateral upward; $C U$, contralateral upward; $C$, contralateral; $C D$, contralateral downward; ID, ipsilateral downward). The top rows indicate the properties of a movement parameter (mean, variance, saccade-reach correlation coefficient), the task condition (EH, eye-hand task; $\mathrm{HO}$, hand-only task; E0, eye-only task), and effectors (saccade and reach). Correlation coefficients between saccade and reach were measured only in the eye-hand task. Each triplet of numbers represents the mean, SE, and $p$ value for the difference in the corresponding property of a movement parameter between all control and inactivation trials. A positive difference in means indicates an increase by inactivation. Triplets with a consistent, significant effect in both monkeys are bold. Triplets with a significant effect only in one monkey are italicized.

reach and saccade amplitudes in each inactivation session and compared them with the mean amplitudes in its counterpart control session (within-pair difference). The significance of the within-pair difference for each pair of an inactivation and a control session was estimated using a permutation task with trial shuffling within the pair (see Materials and Methods). In Monkey $\mathrm{Y}$, the reach amplitude for the contralateral target was significantly reduced by inactivation in 6 of 7 pairs and the saccade amplitude in 4 of 7 pairs (Fig. 2b). Similarly, in Monkey G, the reach amplitude reduction was significant in 3 of 6 pairs, and the saccade amplitude in 4 of 6 pairs. (Fig. $2 b$ ).

Furthermore, we computed the mean reach and saccade amplitude for the contralateral target in each session (within-session mean) and compared the two pools of within-session means, each for control and inactivation respectively. When the two pools were treated as matched samples, both reach and saccade amplitude in the eye-hand task was significantly reduced by inactivation in Monkey Y (WSRT, $p=0.008$ for reaches; $p=0.023$ for saccades) and Monkey $\mathrm{G}$ ( $p=0.031$ for reaches; $p=0.015$ for saccades). However, when the two pools were treated as independent samples, the significance level for the differences between the two pools were weakened. The reach amplitudes were significantly different between the two within-session pools in Monkey $\mathrm{Y}$ (WRST, $p=0.0006$ ), and marginally different for $\mathrm{G}$ ( $p=$ $0.066)$. The saccade amplitudes were marginally different in Monkey Y $(p=0.064)$, and significantly different in Monkey G $(p=0.021)$. Despite the weaker significance levels, the latter test results are still in line with the global effect that PRR inactivation reduced both reach and saccade amplitudes for the contralateral target in the eye-hand task. The reduction of saccade amplitudes in coordinated movements but not in single-effector movements (Fig. 2a) suggests that the reach target representation in PRR affects the spatial target of saccades if the saccades are naturally planned together with reaches and there is a context-dependent coupling during eye-hand coordination.

\section{Effects of PRR inactivation on temporal} eye-hand coordination

To investigate the effect of PRR inactivation on temporal aspects of coordinated reaches and saccades, we examined reach and saccade reaction times (i.e., movement onset time in response to the go cue) in the eye-hand task. Similar to the amplitude anal- 
Table 3. The reach and saccade reaction times in the eye-hand task

\begin{tabular}{|c|c|c|c|c|}
\hline \multirow[b]{2}{*}{ Monkey } & \multicolumn{2}{|c|}{$\begin{array}{l}\text { Reach reaction time } \\
(\mathrm{ms}, \text { mean } \pm S \mathrm{SD})\end{array}$} & \multicolumn{2}{|c|}{$\begin{array}{l}\text { Saccade reaction time } \\
\text { (ms, mean } \pm S D)\end{array}$} \\
\hline & Control & Inactivation & Control & Inactivation \\
\hline \multicolumn{5}{|l|}{ Y } \\
\hline I & $268 \pm 3.3$ & $271 \pm 2.5$ & $220 \pm 2.1$ & $223 \pm 3.4$ \\
\hline IU & $300 \pm 3.1$ & $296 \pm 2.8$ & $219 \pm 2.4$ & $222 \pm 4.2$ \\
\hline CU & $302 \pm 2.6$ & $302 \pm 2.3$ & $218 \pm 3.6$ & $210 \pm 2.5$ \\
\hline$c$ & $289 \pm 3.0$ & $305 \pm 5.4$ & $220 \pm 4.9$ & $237 \pm 6.1$ \\
\hline$C D$ & $256 \pm 2.5$ & $269 \pm 4.5$ & $396 \pm 7.5$ & $378 \pm 8.6$ \\
\hline ID & $250 \pm 6.1$ & $252 \pm 2.1$ & $312 \pm 5.2$ & $290 \pm 4.8$ \\
\hline \multicolumn{5}{|c|}{$2 .-5$} \\
\hline I & $368 \pm 3.8$ & $358 \pm 3.2$ & $245 \pm 2.8$ & $221 \pm 2.7$ \\
\hline IU & $400 \pm 9.6$ & $427 \pm 14.5$ & $229 \pm 4.4$ & $230 \pm 3.9$ \\
\hline CU & $438 \pm 10.9$ & $433 \pm 8.7$ & $282 \pm 6.7$ & $273 \pm 6.5$ \\
\hline C & $412 \pm 3.6$ & $388 \pm 4.3$ & $268 \pm 2.9$ & $252 \pm 2.3$ \\
\hline$C D$ & $409 \pm 8.0$ & $441 \pm 17.5$ & $283 \pm 5.5$ & $314 \pm 7.2$ \\
\hline ID & $467 \pm 16.7$ & $482 \pm 17.1$ & $255 \pm 5.3$ & $250 \pm 5.0$ \\
\hline
\end{tabular}

The means were computed across all control versus inactivation trials, for each target and each monkey. Means with a significant difference between control and inactivation (permutation test, $p<0.05$ corrected for multiple comparison; see Table 2 for the statistical tests on the difference) were italicized.

ysis described above, we first pooled all control and inactivation trials respectively, and assessed the significance of the difference in their global means using a permutation test (trial reshuffling between the two global pools). The mean reaction time of reaches in the eye-hand task significantly increased following PRR inactivation in two of six targets in Monkey $\mathrm{Y}$, but decreased in one target in Monkey G (Tables 2, 3). The mean reaction time of saccades in the eye-hand task significantly increased for one target but significantly decreased for another target in Monkey Y. In Monkey G, the mean saccade reaction time significantly increased for one target, but significantly decreased for two other targets. However, these targets with significant effects are not overlapping between the two monkeys. Thus, no unifying pattern could be found.

Despite the lack of a consistent pattern in the changes of the mean reaction time of each effector, their coordination might have changed due to the inactivation. To test this idea, we examined a trial-by-trial correlation coefficient between the reach and saccade reaction times in the eye-hand task. Similar to previous reports, we found positive trial-by-trial correlations in the reaction times when all control trials were pooled together for each target location (Fig. 3a). These correlation coefficients from control trials were compared with those from inactivation trials to assess the inactivation effect on temporal coupling between the two effectors (Fig. $3 a, b$ ). As depicted in Figure $3 b$, the reaction time correlation was significantly reduced by inactivation for the contralateral target (permutation test, $p=0.002$; Table 2) and ipsilateral upward target $(p=0.007)$ in Monkey Y. In Monkey G, the correlation significantly decreased only in the contralateral target $(p=0.006)$. Therefore, PRR inactivation decreased the temporal correlation between a coordinated reach and a saccade to the contralateral target in both monkeys.

To examine the robustness of this effect for the contralateral target, we computed a reaction time correlation coefficient within each inactivation session, and compared it with the correlation coefficient from its counterpart control session. A significant reduction by inactivation was observed in 3 of 7 sessions in Monkey Y, and 2 of 6 sessions in Monkey G (Fig. $3 c$; permutation test). The correlation coefficients showed a large variance across sessions even within the same conditions (mean $\pm \mathrm{SD}$ of the correlation coefficient: $0.39 \pm 0.178$ for Monkey Y control, $0.07 \pm 0.1778$ for Monkey $Y$ inactivation; $0.55 \pm 0.397$ for Mon- key G control, and $0.40 \pm 0.218$ for Monkey G inactivation), partly because of relatively small numbers of trials in some sessions (range, 8-266; Table 1), in which a few outliers could strongly influence the Pearson correlation coefficients. Nevertheless, the two pools of within-session correlation coefficients, each for control and inactivation respectively, showed a significant difference in a matched sample test for both Monkeys Y (WSRT $p=0.023)$ and $\mathrm{G}(p=0.047)$. In an independent sample test, the correlation difference was significant in Monkey Y (WRST $p=$ $0.019)$, and marginal for Monkey $G(p=0.066)$. To alleviate the issue of different trial numbers among sessions, we also computed a normalized correlation coefficient for each session (see Materials and Methods). After normalization, the correlation difference between the two pools was significant in both matched and independent sample tests in both monkeys (WSRT $p=0.039$ for Monkey Y, $p=0.031$ for Monkey G; WRST $p=0.036$ for Monkey Y, $p=0.015$ for Monkey G).

Together, we conclude that temporal coupling between a spatially coordinated reach and a saccade deteriorated specifically for the target location for which the coordinated reach and saccade amplitudes also decreased due to PRR inactivation.

\section{Effects of PRR inactivation on other spatial and temporal parameters}

We examined inactivation effects on other spatial and temporal parameters including the amplitude variance, amplitude correlation between saccades and reaches, reaction time variance, mean movement duration, movement duration variance, and movement duration correlation between saccades and reaches. We found that inactivation decreased the mean reach movement duration for the contralateral and ipsilateral targets in the eye-hand task for both monkeys, corresponding to the shortened reach amplitude. Except for this effect, no other parameters in the eyehand task showed a significant effect that is consistent between the two monkeys (Table 2).

\section{Discussion}

To understand the neural mechanisms underlying eye-hand coordination, we inactivated PRR, a reach control area in PPC, and examined its effects on the spatial and temporal coordination of reaches and saccades. We found that PRR inactivation changed the concurrently executed reach and saccade endpoints in a congruent manner, while decoupling their reaction times. These results indicate that both spatial and temporal eye-hand coordination relies on PRR.

\section{Spatial eye-hand coordination}

The neurons in PRR are selectively more active during the planning and execution of reaches as opposed to saccades, and their activity is modulated by the planned reach target location. This neural property indicates that PRR represents reach target information and thus is involved in controlling reach endpoints. Consistent with this idea, we previously found that PRR inactivation shortens movement amplitudes, i.e., hypometria, for reaches but not saccades when each effector moves alone (Hwang et al., 2012). In the current study, we found that for concurrently executed reaches and saccades, PRR inactivation induced significant hypometria for both reaches and saccades in a congruent manner. The hypometric saccades, in the presence of concurrent reaches, indicate that the spatial eye-hand coordination is not driven solely by common inputs to independent reach and saccade pathways. The result rather shows that the saccade control pathway modified the saccade target information according to 
a

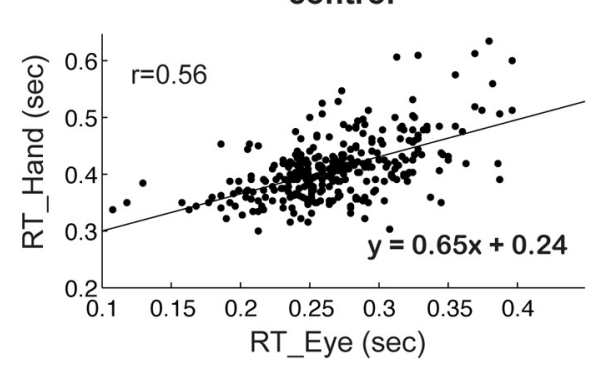

inactivation

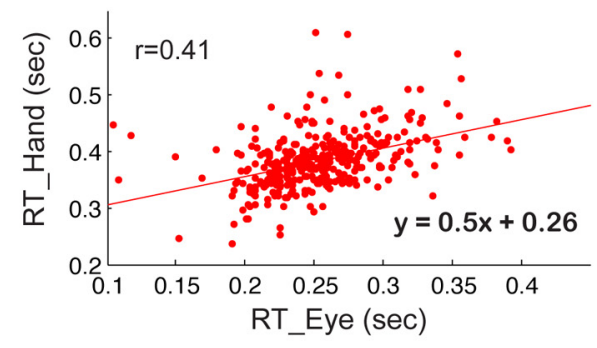

C

monkey $Y$

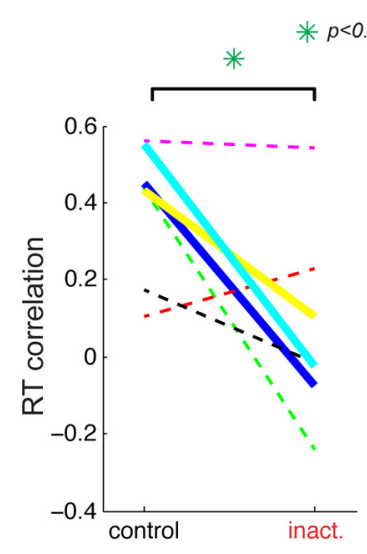

monkey G

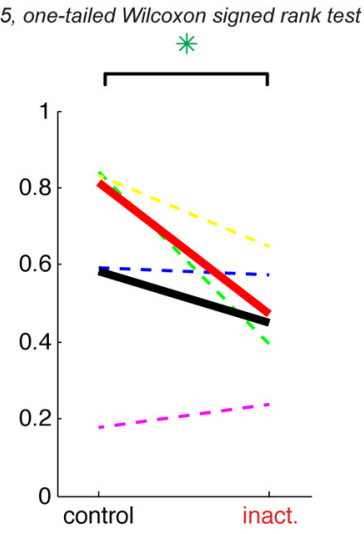

b

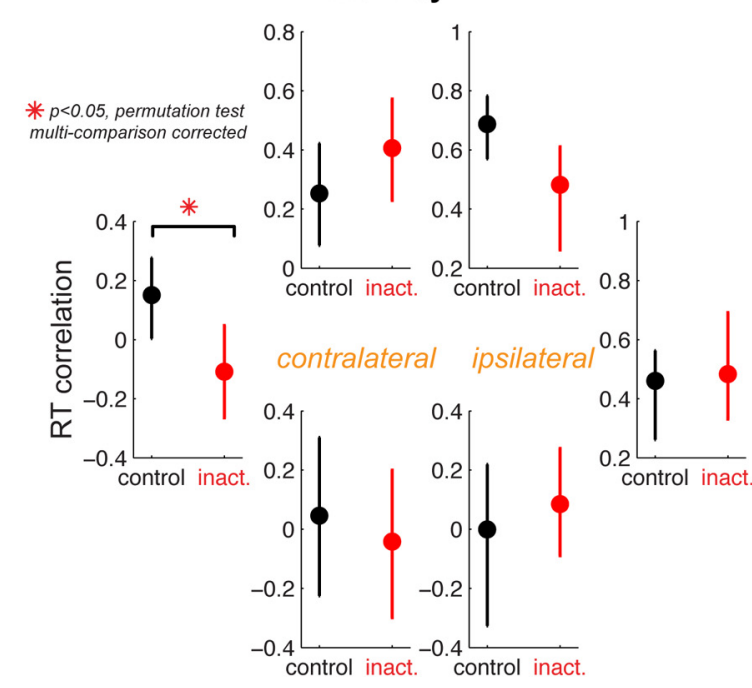

monkey G

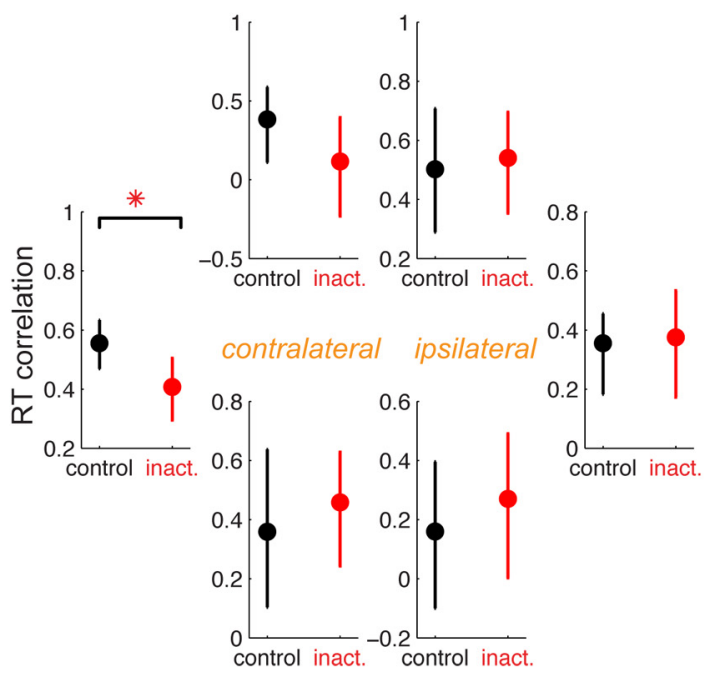

Figure 3. PRR inactivation weakens the temporal correlation between spatially coordinated reaches and saccades. $\boldsymbol{a}$, Saccade reaction times versus reach reaction times (RTs) for the contralateral target in the eye- hand task for all control versus inactivation trials. Each dot represents a single trial, and the lines are the linear regression. $\boldsymbol{b}$, The mean and its $95 \%$ confidence interval of the correlation coefficient between saccade and reach RTs of all control versus inactivation trials for each of the six target locations. c, Within-session RT correlation coefficients for the contralateral target, in control versus inactivation sessions. Each pair of a control and an inactivation session is connected with a solid line if their coefficients are significantly different ( $p<0.05$ ), and with a dashed line otherwise.

the distorted reach target information in PRR (and/or its downstream areas). In other words, spatial information appears to transmit from reach to saccade pathways.

It is also noteworthy that the presence of concurrent saccades lessened the reach amplitude reduction under PRR inactivation (Fig. $2 a$; eye-hand vs hand-only). That is, the planning of concurrent saccades compensated for the distorted reach target information under PRR inactivation, suggesting spatial information transmission from the saccade control pathway to the reach control pathway. Together, these findings suggest that the spatial eye-hand coordination relies on, in part, bidirectional interaction between the reach and saccade control pathways. Thus, in terms of the two hypotheses for eye-hand coordination, interpathway communication or common external inputs, the results support the former hypothesis.

\section{Temporal eye-hand coordination}

PRR inactivation weakened the strength of the trial-by-trial correlation between coordinated reach and saccade reaction times. The deteriorated correlation demonstrates that a tight temporal coupling requires an intact PRR. However, this result alone cannot conclusively reject or accept any mechanism by which PRR may contribute to temporal coupling. For instance, if temporal coupling results from common movement trigger signals to both pathways and the reach pathway cannot respond reliably to the common signals because of inactivation, temporal decoupling would occur. Similarly, temporal decoupling could occur if the saccade control pathway sends its temporal information to PRR (and/or to upstream areas) or the temporal correlations involve bidirectional interaction between PRR and eye control areas, because inactivated PRR may not properly function to coordinate 
with the intact eye controllers. Thus, although PRR seems necessary for the temporal coupling of a reach and a saccade, the exact mechanism cannot be determined based on the results of this study.

However, our results can be informative in light of other previous findings. Yttri et al. (2013) found that LIP inactivation increases the reaction times of saccades but not reaches, when each effector moves alone, but it increases the reaction times of both saccades and reaches, when both effectors move concurrently. This result suggests that the reach control pathway adjusted reach onset time based on the saccade onset time that was delayed due to LIP inactivation. Hence, it is unlikely that temporal coordination results solely from common inputs to two independent pathways. Instead, LIP (and/or its downstream areas) in the saccade control pathway may transmit the saccade onset time information to the reach control pathway. In parallel, Dean et al. (2012) found that a distinct cell assembly in LIP encodes the reaction time, specifically for concurrently executed saccades and reaches. This cell assembly is coherent with the beta-band LFPs which also predict the reaction time of concurrent saccades and reaches in both LIP and PRR, suggesting that coherent spiking activity in the two areas may control reaches and saccades together (Dean et al., 2012; Hagan et al., 2012). Together, one emerging hypothesis is that LIP (and/or its downstream areas) transmits the saccade onset information to PRR (and/or its upstream areas) to generate coherent spiking activity in LIP and PRR, which coordinates temporal coupling. In this hypothesis, our result suggests that inactivated PRR cannot generate spiking activity that is coherent with LIP spiking activity, decoupling the reaction times of saccades and reaches.

\section{Comparison to the other PRR inactivation study}

A recent study by Yttri et al. (2014) also examined the effects of PRR inactivation on eye-hand coordination. Their results differ from ours in several ways. First, their PRR inactivation did not cause hypometria in reaches. Second, their inactivation significantly increased reaction times selectively for reaches whether or not a reach was accompanied by a saccade. Finally, they did not find a significant change in a trial-by-trial reaction time correlation. These discrepancies call for a careful comparison between the two studies.

Perhaps, the most striking difference is in the anatomical locations of inactivation sites. PRR is a functionally defined region, including multiple reach selective cortical areas in PPC. These areas encompass the dorsal aspect of $\mathrm{PO}$ including V6A and the posterior aspect of the medial bank of IPS including MIP and extending anteriorly in the IPS into area 5V (Andersen et al., 2014). The injection sites in Yttri et al. (2013) were more posterior in the IPS and included areas V6a and MIP, whereas our injection sites were more anterior in IPS and included MIP and area $5 \mathrm{~V}$. Thus, although neurons in both inactivated areas are reach selective, their functional roles might differ. For instance, the increase in reach reaction time from Yttri et al., 2014 may be due to involvement of area V6a.

Second, the lack of consistent effects on the mean reaction times in our study might be related to more lenient temporal constraints. In our task, there was virtually no reaction time constraint because the animals were allowed to initiate movements in up to $2 \mathrm{~s}$ after the go cue. In contrast, in Yttri et al. (2013), the movement initiation was enforced to be within $0.5 \mathrm{~s}$ for saccades, and $0.75 \mathrm{~s}$ for reaches. In addition, the delay period varied from 1.0 and $1.3 \mathrm{~s}$ in our study, whereas it was $1.0-1.6 \mathrm{~s}$ in their study. The smaller variability in our delay periods might have allowed the monkeys to anticipate rather than react to the go cue, leading to more variable reaction times.

Thirdly, the concurrent reaches and saccades naturally arose in our study, whereas they were specifically required in Yttri et al. (2013). Perhaps, for generating saccades that naturally accompany reaches, the role of interaction between the saccade and reach pathways may increase, relative to the role of common inputs to the two pathways. If so, PRR inactivation could have a larger effect on the naturally occurring temporal coordination.

Finally, the injections used by Yttri et al. (2013) were of much smaller volume and thus may not have inactivated sufficient PRR tissue to produce measurable effects. This possibility is suggested by the fact that the one deficit they did see, increased reaction time, was very small $(6.8 \mathrm{~ms})$ and was found after combining data from a large number of injections (28).

The lack of effects on eye-hand coordination found by Yttri et al. (2013) led to their very strong statement that PRR does not play a role in coordinating eye-hand movements. On the other hand, our findings of the transfer of spatial information and reduced temporal correlations indicate that eye-hand coordination does rely on PRR. Furthermore, our finding that the amplitude of saccades is affected for eye-hand movements, but not eye movements alone, indicates that the spatial coupling between the eye and hand systems is dynamic and context-dependent.

\section{Comparison with human studies}

Studies of human subjects have revealed two clusters of reach related areas in the PPC. One is located medial-posterior in the precuneus, parietal-occipital junction, and superior parietal occipital cortex and the other more lateral-anterior centered on the medial bank and middle portion of the IPS (Vesia and Crawford, 2012; Andersen et al., 2014). Vesia and Crawford (2012) proposed that the more medial site is for uncoupling reaches and saccades and the more lateral site for coupling them.

The current study in monkeys has similarities and differences with the human data. Inactivation of PRR interferes with the temporal coupling of reaches and saccades and affected concurrently executed reach and saccade endpoints, suggesting that it may be similar to the lateral cluster of humans (Vesia et al., 2010). However, inactivation of PRR produces optic ataxia, which has been proposed to be a deficit in uncoupling reaches and saccades and is often attributed to the more medial cluster (Hwang et al., 2012; Vesia et al., 2012; Andersen et al., 2014). Overall more study is required to determine potential homologies or differences in organization of eye-hand movement circuits in the two primate species.

\section{References}

Andersen RA, Cui H (2009) Intention, action planning, and decision making in parietal-frontal circuits. Neuron 63:568-583. CrossRef Medline

Andersen RA, Andersen KN, Hwang EJ, Hauschild M (2014) Optic ataxia: from Balint's syndrome to the parietal reach region. Neuron 81:967-983. CrossRef Medline

Battaglia-Mayer A, Archambault PS, Caminiti R (2006) The cortical network for eye-hand coordination and its relevance to understanding motor disorders of parietal patients. Neuropsychologia 44:2607-2620. CrossRef Medline

Carey DP (2000) Eye-hand coordination: eye to hand or hand to eye? Curr Biol 10:R416-R419. CrossRef Medline

Crawford JD, Medendorp WP, Marotta JJ (2004) Spatial transformations for eye-hand coordination. J Neurophysiol 92:10-19. CrossRef Medline

Culham JC, Cavina-Pratesi C, Singhal A (2006) The role of parietal cortex in visuomotor control: what have we learned from neuroimaging? Neuropsychologia 44:2668-2684. CrossRef Medline

Dean HL, Martí D, Tsui E, Rinzel J, Pesaran B (2011) Reaction time corre- 
lations during eye-hand coordination: behavior and modeling. J Neurosci 31:2399-2412. CrossRef Medline

Dean HL, Hagan MA, Pesaran B (2012) Only coherent spiking in posterior parietal cortex coordinates looking and reaching. Neuron 73:829-841. CrossRef Medline

Enright JT (1995) The non-visual impact of eye orientation on eye-hand coordination. Vis Res 35:1611-1618. CrossRef Medline

Filimon F (2010) Human cortical control of hand movements: parietofrontal networks for reaching, grasping, and pointing. Neuroscientist 16:388407. CrossRef Medline

Flanagan JR, Terao Y, Johansson RS (2008) Gaze behavior when reaching to remembered targets. J Neurophysiol 100:1533-1543. CrossRef Medline

Frens MA, Erkelens CJ (1991) Coordination of hand movements and saccades: evidence for a common and a separate pathway. Exp Brain Res 85:682-690. Medline

Gaveau V, Pélisson D, Blangero A, Urquizar C, Prablanc C, Vighetto A, Pisella L (2008) Saccade control and eye-hand coordination in optic ataxia. Neuropsychologia 46:475-486. CrossRef Medline

Hagan MA, Dean HL, Pesaran B (2012) Spike-field activity in parietal area LIP during coordinated reach and saccade movements. J Neurophysiol 107:1275-1290. CrossRef Medline

Hayhoe MM, Shrivastava A, Mruczek R, Pelz JB (2003) Visual memory and motor planning in a natural task. J Vis 3(1):6 49-63. CrossRef Medline

Henriques DY, Crawford JD (2000) Direction-dependent distortions of retinocentric space in the visuomotor transformation for pointing. Exp Brain Res 132:179-194. CrossRef Medline

Hwang EJ, Hauschild M, Wilke M, Andersen RA (2012) Inactivation of the parietal reach region causes optic ataxia, impairing reaches but not saccades. Neuron 76:1021-1029. CrossRef Medline

Johansson RS, Westling G, Bäckström A, Flanagan JR (2001) Eye-hand coordination in object manipulation. J Neurosci 21:6917-6932. Medline
Kato T, Fukuda T (2002) Visual search strategies of baseball batters: eye movements during the preparatory phase of batting. Percept Mot Skills 94:380-386. CrossRef Medline

Ko HK, Poletti M, Rucci M (2010) Microsaccades precisely relocate gaze in a high visual acuity task. Nat Neurosci 13:1549-1553. CrossRef Medline

Konen CS, Mruczek RE, Montoya JL, Kastner S (2013) Functional organization of human posterior parietal cortex: grasping- and reaching-related activations relative to topographically organized cortex. J Neurophysiol 109:2897-2908. CrossRef Medline

Sailer U, Eggert T, Ditterich J, Straube A (2000) Spatial and temporal aspects of eye-hand coordination across different tasks. Exp Brain Res 134:163173. CrossRef Medline

Snyder LH, Batista AP, Andersen RA (1997) Coding of intention in the posterior parietal cortex. Nature 386:167-170. CrossRef Medline

Song JH, Takahashi N, McPeek RM (2008) Target selection for visually guided reaching in macaque. J Neurophysiol 99:14-24. CrossRef Medline

Van Donkelaar P, Lee JH, Drew AS (2000) Transcranial magnetic stimulation disrupts eye-hand interactions in the posterior parietal cortex. J Neurophysiol 84:1677-1680. Medline

Vesia M, Crawford JD (2012) Specialization of reach function in human posterior parietal cortex. Exp Brain Res 221:1-18. CrossRef Medline

Vesia M, Prime SL, Yan X, Sergio LE, Crawford JD (2010) Specificity of human parietal saccade and reach regions during transcranial magnetic stimulation. J Neurosci 30:13053-13065. CrossRef Medline

Yttri EA, Liu Y, Snyder LH (2013) Lesions of cortical area LIP affect reach onset only when the reach is accompanied by a saccade, revealing an active eye-hand coordination circuit. Proc Natl Acad Sci U S A 110:2371-2376. CrossRef Medline

Yttri EA, Wang C, Liu Y, Snyder LH (2014) The parietal reach region is limb specific and not involved in eye-hand coordination. J Neurophysiol 111: 520-532. CrossRef Medline 